\title{
A NOTE ON THE LIMITING WEAK-TYPE BEHAVIOR FOR MAXIMAL OPERATORS
}

\author{
JIAXIN HU AND XUEPING HUANG \\ (Communicated by Juha M. Heinonen)
}

\begin{abstract}
We study the following open question raised by Janakiraman in (2006): for $f \in L^{1}\left(\mathbb{R}^{n}\right) \cap L^{\infty}\left(\mathbb{R}^{n}\right)$ and $\lambda>0$, what is the limiting behavior of$$
\left[m\left(\left\{x \in \mathbb{R}^{n}: M\left(|f|^{p}\right)(x)>\lambda\right\}\right)\right]^{1 / p}
$$

as $p \rightarrow \infty$ ? In this note, we give a complete answer to this question.

\section{INTRODUCTION}

Denote by $m$ the Lebesgue measure. Let $M$ be the Hardy-Littlewood maximal operator defined by

$$
M(f)(x)=\sup _{r>0} \frac{1}{m(B(x, r))} \int_{B(x, r)}|f(y)| d y \quad\left(x \in \mathbb{R}^{n}\right),
$$

where $B(x, r)$ is a (closed or open) ball centered at $x$ with radius $r$. The maximal function theorem [4] reads that, for any $f \in L^{1}\left(\mathbb{R}^{n}\right)$ and $\lambda>0$,

$$
m\left(\left\{x \in \mathbb{R}^{n}: M(f)(x)>\lambda\right\}\right) \leq \frac{c_{1}}{\lambda} \int_{\mathbb{R}^{n}}|f| d m,
$$

where $c_{1}>0$ is independent of $\lambda$ and $f$. See also [1, Theorem 2.2] for doubling metric measure spaces. The reverse to (1.1) also holds: for $f \in L^{1}\left(\mathbb{R}^{n}\right)$ and $\lambda>0$, there exist $c, \bar{c}>0$ independent of $f$ and $\lambda$ such that

$$
m\left(\left\{x \in \mathbb{R}^{n}: M(f)(x)>\lambda\right\}\right) \geq \frac{c}{\lambda} \int_{\left\{x \in \mathbb{R}^{n}:|f(x)|>\lambda / \bar{c}\right\}}|f| d m .
$$

The reader may find the sketched proof in [3, p. 43]. The formulae (1.1) and (1.2) give the upper and lower bounds of

$$
\lambda m\left(\left\{x \in \mathbb{R}^{n}: M(f)(x)>\lambda\right\}\right) .
$$

Recently, Janakiraman [2] has obtained, among other things, the following interesting result about the limiting behavior of (1.3):

$$
\lim _{\lambda \rightarrow 0} \lambda m\left(\left\{x \in \mathbb{R}^{n}: M(f)(x)>\lambda\right\}\right)=\|f\|_{1}, \quad f \in L^{1}\left(\mathbb{R}^{n}\right) .
$$

Received by the editors September 3, 2006.

2000 Mathematics Subject Classification. Primary 42B25.

(C) 2008 American Mathematical Society Reverts to public domain 28 years from publication 
Janakiraman further guessed the following ([2, p. 1947]):

$$
\lim _{p \rightarrow \infty}\left[m\left(\left\{x \in \mathbb{R}^{n}: M\left(|f|^{p}\right)(x)>\lambda\right\}\right)\right]^{1 / p}
$$

$$
= \begin{cases}\|f\|_{\infty}, & \text { if } m(\{|f|>1\})>0, \\ 0, & \text { if }|f|<1 \text { a.e. } \\ \chi_{(0,1)}(\lambda), & \text { if } m(\{|f|=1\})>0,\end{cases}
$$

where $\chi_{A}$ is the characteristic function for a measurable set $A$, that is, $\chi_{A}(x)=1$ for $x \in A$, and $\chi_{A}(x)=0$ otherwise.

In this note, we will prove that the first equality in $(\mathbf{A})$ is true; see Section 2. However, the other two inequalities are not true. Instead, the third equality in (A) is true under an additional assumption; see Theorem 3.1. For the second equality in (A), we give a counterexample; see Example 4.3. For this case, what we have obtained is that: for $f \in L^{1}\left(\mathbb{R}^{n}\right) \cap L^{\infty}\left(\mathbb{R}^{n}\right)$ with $\|f\|_{\infty}=1$,

$$
\begin{aligned}
\varlimsup_{p \rightarrow \infty}\left[m \left(\left\{x \in \mathbb{R}^{n}: M\left(|f|^{p}\right)(x)\right.\right.\right. & >\lambda\})]^{1 / p} \\
& =\varlimsup_{p \rightarrow \infty}\left[m\left(\left\{x \in \mathbb{R}^{n}:|f|^{p}(x)>\lambda\right\}\right)\right]^{1 / p},
\end{aligned}
$$

and

$$
\begin{aligned}
\varliminf_{p \rightarrow \infty}\left[m \left(\left\{x \in \mathbb{R}^{n}: M\left(|f|^{p}\right)(x)\right.\right.\right. & >\lambda\})]^{1 / p} \\
& =\varliminf_{p \rightarrow \infty}\left[m\left(\left\{x \in \mathbb{R}^{n}:|f|^{p}(x)>\lambda\right\}\right)\right]^{1 / p} .
\end{aligned}
$$

Finally, we discuss formula (1.4) for a general measure. We will give an example, showing that if the Lebesgue measure $m$ is replaced by a doubling measure $\mu$, then (1.4) fails; see Section 5.

\section{Proof of the first Equality in (A)}

It is known that, for $f \in L^{1}\left(\mathbb{R}^{n}\right) \cap L^{\infty}\left(\mathbb{R}^{n}\right)$, the function $f \in L^{p}\left(\mathbb{R}^{n}\right)$ for $1<$ $p<\infty$, and $\lim _{p \rightarrow \infty}\|f\|_{p}=\|f\|_{\infty}$.

Proposition 2.1. For $f \in L^{1}\left(\mathbb{R}^{n}\right) \cap L^{\infty}\left(\mathbb{R}^{n}\right)$ and $\lambda>0$,

$$
\varlimsup_{p \rightarrow \infty}\left[m\left(\left\{x \in \mathbb{R}^{n}: M\left(|f|^{p}\right)(x)>\lambda\right\}\right)\right]^{1 / p} \leq\|f\|_{\infty} .
$$

Proof. Note that $|f|^{p} \in L^{1}\left(\mathbb{R}^{n}\right)$, and by (1.1),

$$
m\left(\left\{x \in \mathbb{R}^{n}: M\left(|f|^{p}\right)(x)>\lambda\right\}\right) \leq \frac{c_{1}}{\lambda} \int_{\mathbb{R}^{n}}|f|^{p} d m .
$$

Therefore,

$$
\begin{aligned}
\varlimsup_{p \rightarrow \infty}\left[m\left(\left\{x \in \mathbb{R}^{n}: M\left(|f|^{p}\right)(x)>\lambda\right\}\right)\right]^{1 / p} & \leq \varlimsup_{p \rightarrow \infty}\left[\frac{c_{1}}{\lambda} \int_{\mathbb{R}^{n}}|f|^{p} d m\right]^{1 / p} \\
& =\|f\|_{\infty} .
\end{aligned}
$$

This completes the proof.

In the rest of this paper, we denote by $\{f>a\}$ the set of all points $x \in \mathbb{R}^{n}$ such that $f(x)>a$. 
Proposition 2.2. Suppose that $f \in L^{1}\left(\mathbb{R}^{n}\right) \cap L^{\infty}\left(\mathbb{R}^{n}\right)$ and $m(\{|f|>1\})>0$. Then

$$
\varliminf_{p \rightarrow \infty}\left[m\left(\left\{x \in \mathbb{R}^{n}: M\left(|f|^{p}\right)(x)>\lambda\right\}\right)\right]^{1 / p} \geq\|f\|_{\infty} .
$$

Proof. Since $|f|^{p} \in L^{1}\left(\mathbb{R}^{n}\right)$, we apply (1.2) for $|f|^{p}$ to obtain that

$$
m\left(\left\{M\left(|f|^{p}\right)>\lambda\right\}\right) \geq \frac{c}{\lambda} \int_{\left\{|f|^{p}>\lambda / \bar{c}\right\}}|f|^{p} d m,
$$

which implies that

$$
\begin{aligned}
\varliminf_{p \rightarrow \infty}\left[m\left(\left\{M\left(|f|^{p}\right)>\lambda\right\}\right)\right]^{1 / p} & \geq \varliminf_{p \rightarrow \infty}\left(\frac{c}{\lambda} \int_{\left\{|f|^{p}>\lambda / \bar{c}\right\}}|f|^{p} d m\right)^{1 / p} \\
& \geq \varliminf_{p \rightarrow \infty}\left(\int_{\left\{|f|^{p}>\lambda / \bar{c}\right\}}|f|^{p} d m\right)^{1 / p} .
\end{aligned}
$$

On the other hand, the inequality $m(\{|f|>1\})>0$ implies that $\|f\|_{\infty}>1$. Thus, for any small $0<\varepsilon<\|f\|_{\infty}-1$, we have

$$
0<m\left(\left\{|f|>\|f\|_{\infty}-\varepsilon\right\}\right)<\infty .
$$

Since $\|f\|_{\infty}-\varepsilon>1$, there exists $p_{\varepsilon}$ such that $\left(\|f\|_{\infty}-\varepsilon\right)^{p_{\varepsilon}}>\lambda / \bar{c}$. This gives that, for $p>p_{\varepsilon}$,

$$
\left\{|f|>\|f\|_{\infty}-\varepsilon\right\} \subseteq\left\{|f|^{p}>\lambda / \bar{c}\right\} .
$$

Therefore, it follows from (2.6) that

$$
\begin{aligned}
\varliminf_{p \rightarrow \infty}\left[m\left(\left\{M\left(|f|^{p}\right)>\lambda\right\}\right)\right]^{1 / p} & \geq \varliminf_{p \rightarrow \infty}\left(\int_{\left\{|f|>\|f\|_{\infty}-\varepsilon\right\}}|f|^{p} d m\right)^{1 / p} \\
& \geq\|f\|_{\infty}-\varepsilon .
\end{aligned}
$$

Hence (2.4) follows by letting $\varepsilon \rightarrow 0^{+}$.

By Propositions 2.1 and 2.2, it follows that, for $f \in L^{1}\left(\mathbb{R}^{n}\right) \cap L^{\infty}\left(\mathbb{R}^{n}\right)$ and $\lambda>0$, if $m(\{|f|>1\})>0$, then

$$
\lim _{p \rightarrow \infty}\left[m\left(\left\{x \in \mathbb{R}^{n}: M\left(|f|^{p}\right)(x)>\lambda\right\}\right)\right]^{1 / p}=\|f\|_{\infty} .
$$

This proves the first equality in $(\mathbf{A})$.

\section{The Third EQUality in (A)}

Theorem 3.1. Let $f \in L^{1}\left(\mathbb{R}^{n}\right) \cap L^{\infty}\left(\mathbb{R}^{n}\right)$ and $\lambda>0$. If $m(\{|f|=1\})>0$ and $\|f\|_{\infty}=1$, then

$$
\lim _{p \rightarrow \infty}\left[m\left(\left\{x \in \mathbb{R}^{n}: M\left(|f|^{p}\right)(x)>\lambda\right\}\right)\right]^{1 / p}=\chi_{(0,1)}(\lambda) .
$$

Proof. Case 1. Let $\lambda \geq 1$. Since $\|f\|_{\infty}=1$, we have $|f| \leq 1$ a.e. Thus

$$
M\left(|f|^{p}\right) \leq\left\||f|^{p}\right\|_{\infty} \leq 1
$$

for any $p>0$. Hence, the Lebesgue measure of the set $\left\{x \in \mathbb{R}^{n}: M\left(|f|^{p}\right)(x)>\lambda\right\}$ is zero, and (3.1) immediately follows for $\lambda \geq 1$. 
Case 2. Let $0<\lambda<1$. Set $A=\left\{x \in \mathbb{R}^{n}:|f(x)|=1\right\}$. By the assumption, we see that $m(A)>0$, and $m(A)<\infty$ for $f \in L^{1}$. Clearly $|f|^{p} \geq \chi_{A}$, and so $M\left(|f|^{p}\right) \geq M\left(\chi_{A}\right)$. Therefore,

$$
\left\{x \in \mathbb{R}^{n}: M\left(\chi_{A}\right)(x)>\lambda\right\} \subseteq\left\{x \in \mathbb{R}^{n}: M\left(|f|^{p}\right)(x)>\lambda\right\} .
$$

Hence,

$$
\begin{aligned}
\varliminf_{p \rightarrow \infty}\left[m \left(\left\{x \in \mathbb{R}^{n}: M\left(|f|^{p}\right)(x)\right.\right.\right. & >\lambda\})]^{1 / p} \\
& \geq \underline{\lim _{p \rightarrow \infty}}\left[m\left(\left\{x \in \mathbb{R}^{n}: M\left(\chi_{A}\right)(x)>\lambda\right\}\right)\right]^{1 / p} .
\end{aligned}
$$

Noting that $0<\lambda<1$, we conclude that

$$
0<m\left(\left\{x \in \mathbb{R}^{n}: M\left(\chi_{A}\right)(x)>\lambda\right\}\right)<\frac{c_{1}}{\lambda} \int_{\mathbb{R}^{n}} \chi_{A} d m=\frac{c_{1}}{\lambda} m(A)<\infty,
$$

which combines with (3.2) to yield that

$$
\varliminf_{p \rightarrow \infty}\left[m\left(\left\{x \in \mathbb{R}^{n}: M\left(|f|^{p}\right)(x)>\lambda\right\}\right)\right]^{1 / p} \geq 1 .
$$

Combining this with (2.1), we obtain the desired proof.

\section{Discussions for the Second equality in (A)}

If $\delta:=\|f\|_{\infty}<1$, then $M\left(|f|^{p}\right) \leq \delta^{p} \rightarrow 0$ as $p \rightarrow \infty$. For any $\lambda>0$, the set

$$
\left\{x \in \mathbb{R}^{n}: M\left(|f|^{p}\right)(x)>\lambda\right\}
$$

is empty for $p$ sufficiently large. Thus, we have

$$
\lim _{p \rightarrow \infty}\left[m\left(\left\{x \in \mathbb{R}^{n}: M\left(|f|^{p}\right)(x)>\lambda\right\}\right)\right]^{1 / p}=0
$$

for any $\lambda>0$. At this time, the second equality in $(\mathbf{A})$ does hold. However, it is possible that $|f|<1$ a.e., but $\|f\|_{\infty}=1$. This situation is more awkward. As we will see, the limit in (4.1) may not exist.

Example 4.1. Let $f=(1-x) \chi_{[0,1]}$ and $0<\lambda<1$. Clearly $|f|<1$ a.e., and $\|f\|_{\infty}=1$ and $f \in L^{1}(\mathbb{R}) \cap L^{\infty}(\mathbb{R})$. Note that, by the Lebesgue differentiation theorem,

$$
\frac{1}{m(B(x, r))} \int_{B(x, r)}|f(y)|^{p} d y \rightarrow|f(x)|^{p}
$$

for almost all $x \in \mathbb{R}^{n}$ as $r \rightarrow 0$, and so, by definition,

$$
M\left(|f|^{p}\right) \geq|f|^{p} \quad \text { a.e. }
$$

Hence

$$
m\left(\left\{x \in \mathbb{R}^{n}: M\left(|f|^{p}\right)(x)>\lambda\right\}\right) \geq m\left(\left\{x \in \mathbb{R}^{n}:|f(x)|^{p}>\lambda\right\}\right)=1-\lambda^{1 / p} .
$$

A simple computation shows that $\lim _{p \rightarrow \infty}\left(1-\lambda^{1 / p}\right)^{1 / p}=1$, and so

$$
\varliminf_{p \rightarrow \infty}\left[m\left(\left\{x \in \mathbb{R}^{n}: M\left(|f|^{p}\right)(x)>\lambda\right\}\right)\right]^{1 / p} \geq 1 .
$$

Actually the reader may verify that, for this function $f$,

$$
\lim _{p \rightarrow \infty}\left[m\left(\left\{x \in \mathbb{R}^{n}: M\left(|f|^{p}\right)(x)>\lambda\right\}\right)\right]^{1 / p}=\chi_{(0,1)}(\lambda) .
$$


Proposition 4.2. For any $f \in L^{1}\left(\mathbb{R}^{n}\right)$ and for any $\lambda>0,0<k<1$,

$$
m(\{M(f)>\lambda\}) \leq \frac{c_{1}}{(1-k) \lambda} \int_{\{|f|>k \lambda\}}|f(x)| d x,
$$

where $c_{1}$ is the same as in (1.1) (independent of $k$ ).

Proof. We divide $|f|$ into two parts: the "good" one, $g:=|f| \chi_{\{|f| \leq k \lambda\}}$, and the "bad" one, $b:=|f| \chi_{\{|f|>k \lambda\}}$. Since $|f|=g+b$, we see that

$$
M(f) \leq M(g)+M(b) \leq k \lambda+M(b) .
$$

Thus $\{M(f)>\lambda\} \subseteq\{M(b)>(1-k) \lambda\}$, and so

$$
\begin{aligned}
m(\{M(f)>\lambda\}) & \leq m(\{M(b)>(1-k) \lambda\}) \\
& \leq \frac{c_{1}}{(1-k) \lambda} \int_{\{|f|>k \lambda\}}|f(x)| d x
\end{aligned}
$$

by virtue of (1.1).

We are now in a position to prove (1.5) and (1.6), by using (4.3).

Proof of (1.5) and (1.6). Let $0<\lambda<1$. Since $M\left(|f|^{p}\right) \geq|f|^{p}$ a.e., it follows that

$$
m\left(\left\{M\left(|f|^{p}\right)>\lambda\right\}\right) \geq m\left(\left\{|f|^{p}>\lambda\right\}\right) .
$$

Therefore,

$$
\begin{gathered}
\varlimsup_{p \rightarrow \infty}\left[m\left(\left\{M\left(|f|^{p}\right)>\lambda\right\}\right)\right]^{1 / p} \geq \varlimsup_{p \rightarrow \infty}\left[m\left(\left\{|f|^{p}>\lambda\right\}\right)\right]^{1 / p}, \\
\varliminf_{p \rightarrow \infty}\left[m\left(\left\{M\left(|f|^{p}\right)>\lambda\right\}\right)\right]^{1 / p} \geq \underset{p \rightarrow \infty}{\lim _{p \rightarrow \infty}\left[m\left(\left\{|f|^{p}>\lambda\right\}\right)\right]^{1 / p} .}
\end{gathered}
$$

On the other hand, as $|f|^{p} \in L^{1}\left(\mathbb{R}^{n}\right)$, we apply (4.3) to $|f|^{p}$, and obtain that, using the assumption that $|f| \leq 1$ a.e.,

$$
\begin{aligned}
m\left(\left\{M\left(|f|^{p}\right)>\lambda\right\}\right) & \leq \frac{c_{1}}{(1-k) \lambda} \int_{\left\{|f|^{p}>k \lambda\right\}}|f(x)|^{p} d x \\
& \leq \frac{c_{1}}{(1-k) \lambda} m\left(\left\{|f|^{p}>k \lambda\right\}\right) .
\end{aligned}
$$

Since $0<\lambda, k<1$, we have

$$
\begin{aligned}
{\left[m\left(\left\{|f|^{p}>k \lambda\right\}\right)\right]^{1 / p} } & =\left[m\left(\left\{|f|^{\frac{p \ln \lambda}{\ln k+\ln \lambda}}>\lambda\right\}\right)\right]^{\frac{\ln k+\ln \lambda}{p \ln \lambda} \times \frac{\ln \lambda}{\ln k+\ln \lambda}} \\
& :=\left[m\left(\left\{|f|^{q}>\lambda\right\}\right)\right]^{\frac{1}{q} \times \frac{\ln \lambda}{\ln k+\ln \lambda}}
\end{aligned}
$$

where $q:=\frac{p \ln \lambda}{\ln k+\ln \lambda}$. This combines with (4.6) to yield that

$$
\left[m\left(\left\{M\left(|f|^{p}\right)>\lambda\right\}\right)\right]^{1 / p} \leq\left(\frac{c_{1}}{(1-k) \lambda}\right)^{1 / p}\left[m\left(\left\{|f|^{q}>\lambda\right\}\right)\right]^{\frac{1}{q} \times \frac{\ln \lambda}{\ln k+\ln \lambda}} .
$$

Taking the lower and upper limits in (4.7), we obtain

$$
\begin{aligned}
& \varlimsup_{p \rightarrow \infty}\left[m\left(\left\{M\left(|f|^{p}\right)>\lambda\right\}\right)\right]^{1 / p} \leq \varlimsup_{q \rightarrow \infty}\left[m\left(\left\{|f|^{q}>\lambda\right\}\right)\right]^{\frac{1}{q} \times \frac{\ln \lambda}{\ln k+\ln \lambda}}, \\
& \varliminf_{p \rightarrow \infty}\left[m\left(\left\{M\left(|f|^{p}\right)>\lambda\right\}\right)\right]^{1 / p} \leq \varliminf_{q \rightarrow \infty}\left[m\left(\left\{|f|^{q}>\lambda\right\}\right)\right]^{\frac{1}{q} \times \frac{\ln \lambda}{\ln k+\ln \lambda}} .
\end{aligned}
$$


Letting $k \rightarrow 1^{-}$and changing $q$ by $p$, we see that

$$
\begin{gathered}
\varlimsup_{p \rightarrow \infty}\left[m\left(\left\{M\left(|f|^{p}\right)>\lambda\right\}\right)\right]^{1 / p} \leq \varlimsup_{p \rightarrow \infty}\left[m\left(\left\{|f|^{p}>\lambda\right\}\right)\right]^{\frac{1}{p}}, \\
\varliminf_{p \rightarrow \infty}\left[m\left(\left\{M\left(|f|^{p}\right)>\lambda\right\}\right)\right]^{1 / p} \leq \varliminf_{p \rightarrow \infty}\left[m\left(\left\{|f|^{p}>\lambda\right\}\right)\right]^{\frac{1}{p}} .
\end{gathered}
$$

Combining this with (4.5), we obtain (1.5) and (1.6).

The following example shows that the limit of $\left[m\left(\left\{M\left(|f|^{p}\right)>\lambda\right\}\right)\right]^{1 / p}$ as $p \rightarrow \infty$ may not exist.

Example 4.3. Consider the function $f$ given by

$$
f=\sum_{n=0}^{\infty} \exp \left(-2^{-n}\right) \chi_{\left(\exp \left(-2^{n}\right), \exp \left(-2^{n-1}\right)\right]} .
$$

Clearly $f \in L^{1}(\mathbb{R}) \cap L^{\infty}(\mathbb{R})$, and $0<f<1$ a.e. and $\|f\|_{\infty}=1$. For $0<\lambda<1$, let

$$
p_{k}=\left(2^{k+1}-1\right) \ln (1 / \lambda) \quad \text { and } \quad q_{k}=\left(2^{k}+1\right) \ln (1 / \lambda) \quad(k \geq 1) .
$$

Note that

$$
\exp \left(-2^{-k}\right)<\lambda^{1 / p_{k}}=\exp \left(-\frac{1}{2^{k+1}-1}\right)<\exp \left(-2^{-k-1}\right),
$$

and so,

$$
m\left(\left\{f^{p_{k}}>\lambda\right\}\right)=\exp \left(-2^{k}\right)
$$

Therefore,

$$
\lim _{p_{k} \rightarrow \infty}\left[m\left(\left\{f^{p_{k}}>\lambda\right\}\right)\right]^{1 / p_{k}}=\lim _{k \rightarrow \infty} \exp \left(\frac{2^{k}}{\left(2^{k+1}-1\right) \ln \lambda}\right)=\exp \left(\frac{1}{2 \ln \lambda}\right) .
$$

A similar computation for $\left\{q_{k}\right\}_{k \geq 1}$ shows that

$$
\lim _{q_{k} \rightarrow \infty}\left[m\left(\left\{f^{q_{k}}>\lambda\right\}\right)\right]^{1 / q_{k}}=\lim _{k \rightarrow \infty} \exp \left(\frac{2^{k}}{\left(2^{k}+1\right) \ln \lambda}\right)=\exp \left(\frac{1}{\ln \lambda}\right) .
$$

By (1.5) and (1.6), we see that the limit of $\left[m\left(\left\{M\left(|f|^{p}\right)>\lambda\right\}\right)\right]^{1 / p}$ as $p \rightarrow \infty$ does not exist.

\section{Discussion For (1.4)}

There is a natural question: if the Lebesgue measure $m$ is replaced by a more general measure $\mu$, say a doubling measure, and $\mathbb{R}^{n}$ replaced by a metric space $X$, does (1.4) still hold? The answer is negative. Here we give an example, showing that the limit of

$$
\lambda \mu(\{x \in X: M(f)(x)>\lambda\})
$$

exists as $\lambda \rightarrow 0$ for a doubling metric measure space $(X, \mu)$, but does not equal $\|f\|_{1}$.

Example 5.1. Let $X=(0, \infty)$ with the Euclidean metric, and let $\mu$ be a measure determined by $d \mu=1_{\{x>0\}} x d x$. It is easy to see that

$$
\mu\left(B\left(x_{0}, r\right)\right)= \begin{cases}2 x_{0} r, & \text { if } x_{0} \geq r>0, \\ \left(x_{0}+r\right)^{2} / 2, & \text { if } r>x_{0}>0 .\end{cases}
$$


Thus, for any $x_{0} \in X$ and $r>0$,

$$
\mu\left(B\left(x_{0}, 2 r\right)\right) \leq 4 \mu\left(B\left(x_{0}, r\right)\right),
$$

showing that $(X, \mu)$ defined as above is a doubling metric measure space.

We claim that for $f \in L^{1}(X, \mu)$,

$$
\lim _{\lambda \rightarrow 0} \lambda \mu(\{x \in X: M(f)(x)>\lambda\})=\frac{1}{4}\|f\|_{1} .
$$

Proof of the claim. For any $\varepsilon>0$, there exists $r_{\varepsilon}>0$ such that

$$
\int_{0}^{r_{\varepsilon}}|f| d \mu>(1-\varepsilon)\|f\|_{1}
$$

for $f \in L^{1}(X, \mu)$. Set

$$
R_{\varepsilon}:=\left(1+\frac{1}{\varepsilon}\right) r_{\varepsilon}
$$

It follows from (5.1) that, for $x_{0}>R_{\varepsilon}$,

$$
\begin{aligned}
(1-\varepsilon) \mu\left(B\left(x_{0}, x_{0}+r_{\varepsilon}\right)\right) & =\frac{1-\varepsilon}{2}\left(2 x_{0}+r_{\varepsilon}\right)^{2}<2 x_{0}^{2}=\mu\left(B\left(x_{0}, x_{0}\right)\right) \\
& <(1+\varepsilon) 2 x_{0}\left(x_{0}-r_{\varepsilon}\right)=(1+\varepsilon) \mu\left(B\left(x_{0}, x_{0}-r_{\varepsilon}\right)\right) .
\end{aligned}
$$

For $\lambda>0$, we divide the set $E_{\lambda}:=\{x \in X: M(f)(x)>\lambda\}$ into three disjoint parts:

$$
\begin{aligned}
& E_{\lambda}^{1}=\left\{x \in E_{\lambda}: x>R_{\varepsilon}, M(f)(x)>\|f\|_{1} / \mu\left(B\left(x, x-r_{\varepsilon}\right)\right)\right\}, \\
& E_{\lambda}^{2}=\left\{x \in E_{\lambda}: x>R_{\varepsilon}, M(f)(x) \leq\|f\|_{1} / \mu\left(B\left(x, x-r_{\varepsilon}\right)\right)\right\}, \\
& E_{\lambda}^{3}=\left\{x \in E_{\lambda}: x \leq R_{\varepsilon}\right\} .
\end{aligned}
$$

First we consider the set $E_{\lambda}^{1}$. For any $x_{0} \in E_{\lambda}^{1}$, we have

$$
\sup _{r>x_{0}-r_{\varepsilon}} \frac{1}{\mu\left(B\left(x_{0}, r\right)\right)} \int_{B\left(x_{0}, r\right)}|f| d \mu \leq \frac{\|f\|_{1}}{\mu\left(B\left(x_{0}, x_{0}-r_{\varepsilon}\right)\right)}<M(f)\left(x_{0}\right) .
$$

Therefore,

$$
\begin{aligned}
M(f)\left(x_{0}\right) & =\sup _{r>0} \frac{1}{\mu\left(B\left(x_{0}, r\right)\right)} \int_{B\left(x_{0}, r\right)}|f| d \mu \\
& =\sup _{0<r \leq x_{0}-r_{\varepsilon}} \frac{1}{\mu\left(B\left(x_{0}, r\right)\right)} \int_{B\left(x_{0}, r\right)}|f| d \mu .
\end{aligned}
$$

Since $M(f)\left(x_{0}\right)>\lambda$, there exists some $r$ with $0<r \leq x_{0}-r_{\varepsilon}$ such that

$$
\frac{1}{\mu\left(B\left(x_{0}, r\right)\right)} \int_{B\left(x_{0}, r\right)}|f| d \mu>\lambda .
$$

From this, we see that

$$
2 r^{2}<2 x_{0} r=\mu\left(B\left(x_{0}, r\right)\right)<\|f\|_{1} / \lambda,
$$

showing that $r$ is bounded from above by $\sqrt{\|f\|_{1} / 2 \lambda}$. Thus the set $E_{\lambda}^{1}$ can be covered by a family of balls with radii uniformly bounded. By the basic covering theorem [1, Theorem 1.16] for the doubling space, there is a countable disjoint subfamily of balls $\left\{B\left(x_{i}, r_{i}\right)\right\}$ such that $E_{\lambda}^{1} \subseteq \bigcup_{i} B\left(x_{i}, 5 r_{i}\right)$. Since $\mu$ is doubling, there exists some $C>0$ such that

$$
\mu\left(B\left(x_{i}, 5 r_{i}\right)\right) \leq C \mu\left(B\left(x_{i}, r_{i}\right)\right) .
$$


Hence, using the fact that $B\left(x_{i}, r_{i}\right) \subset\left[r_{\varepsilon}, \infty\right)$ for each $i$,

$$
\begin{aligned}
\mu\left(E_{\lambda}^{1}\right) & \leq \sum_{i} \mu\left(B\left(x_{i}, 5 r_{i}\right)\right) \leq C \sum_{i} \mu\left(B\left(x_{i}, r_{i}\right)\right) \\
& <C \sum_{i} \frac{1}{\lambda} \int_{B\left(x_{i}, r_{i}\right)}|f| d \mu \leq \frac{C}{\lambda} \int_{r_{\varepsilon}}^{\infty}|f| d \mu \\
& =\frac{C}{\lambda}\left(\|f\|_{1}-\int_{0}^{r_{\varepsilon}}|f| d \mu\right) \leq \frac{C \varepsilon}{\lambda}\|f\|_{1} .
\end{aligned}
$$

Next we consider the set $E_{\lambda}^{2}$. By (5.4), we see that

$$
\begin{aligned}
E_{\lambda}^{2} & \subseteq\left\{x \in X: x>R_{\varepsilon}, \lambda \leq\|f\|_{1} / \mu\left(B\left(x, x-r_{\varepsilon}\right)\right)\right\} \\
& \subseteq\left\{x \in X: x>R_{\varepsilon}, \mu(B(x, x)) \leq(1+\varepsilon)\|f\|_{1} / \lambda\right\} \\
& =\left(R_{\varepsilon},\left[(1+\varepsilon)\|f\|_{1} /(2 \lambda)\right]^{1 / 2}\right):=I_{1} .
\end{aligned}
$$

It follows that

$$
\mu\left(E_{\lambda}^{2}\right) \leq \mu\left(I_{1}\right)=(1+\varepsilon)\|f\|_{1} /(4 \lambda)-R_{\varepsilon}^{2} / 2 .
$$

Combining (5.6) and (5.7), we obtain

$$
\begin{aligned}
\lambda \mu(\{M(f)>\lambda\}) & =\lambda\left(\mu\left(E_{\lambda}^{1}\right)+\mu\left(E_{\lambda}^{2}\right)+\mu\left(E_{\lambda}^{3}\right)\right) \\
& \leq C \varepsilon\|f\|_{1}+(1+\varepsilon)\|f\|_{1} / 4-\lambda R_{\varepsilon}^{2} / 2+\lambda \mu\left(B\left(0, R_{\varepsilon}\right)\right) \\
& =C \varepsilon\|f\|_{1}+(1+\varepsilon)\|f\|_{1} / 4 .
\end{aligned}
$$

In order to obtain the inverse inequality, we observe that for $x_{0} \in E_{\lambda}^{1} \cup E_{\lambda}^{2}$,

$$
\begin{aligned}
M(f)\left(x_{0}\right) & \geq \frac{1}{\mu\left(B\left(x_{0}, x_{0}\right)\right)} \int_{B\left(x_{0}, x_{0}\right)}|f| d \mu=\frac{1}{\mu\left(B\left(x_{0}, x_{0}\right)\right)} \int_{0}^{2 x_{0}}|f| d \mu \\
& \geq \frac{1}{\mu\left(B\left(x_{0}, x_{0}\right)\right)} \int_{0}^{r_{\varepsilon}}|f| d \mu>\frac{(1-\varepsilon)\|f\|_{1}}{\mu\left(B\left(x_{0}, x_{0}\right)\right)}
\end{aligned}
$$

by using the fact that $x_{0}>R_{\varepsilon}>r_{\varepsilon}$. This implies that

$$
\begin{aligned}
E_{\lambda}^{1} \cup E_{\lambda}^{2} & =\left\{x \in X: x>R_{\varepsilon}, M(f)(x)>\lambda\right\} \\
& \supseteq\left\{x \in X: x>R_{\varepsilon},(1-\varepsilon)\|f\|_{1} / \mu(B(x, x))>\lambda\right\} \\
& =\left(R_{\varepsilon},\left[(1-\varepsilon)\|f\|_{1} /(2 \lambda)\right]^{1 / 2}\right):=I_{2} .
\end{aligned}
$$

Therefore,

$$
\begin{aligned}
\lambda \mu(\{M(f)>\lambda\}) & \geq \lambda \mu\left(E_{\lambda}^{1} \cup E_{\lambda}^{2}\right) \\
& \geq \lambda \mu\left(I_{2}\right)=(1-\varepsilon)\|f\|_{1} / 4-\lambda R_{\varepsilon}^{2} / 2 .
\end{aligned}
$$

Finally, letting $\lambda \rightarrow 0$ in both (5.8) and (5.9) and noting that $\varepsilon$ is arbitrary, we arrive at (5.3). This finishes the proof of our claim.

One may further consider the measure $\mu$ on $\mathbb{R}^{n}$ where $d \mu=|x|^{\alpha} d x$ for $\alpha>0$. It can be proven that for suitable $\alpha>0$, the limit

$$
\lim _{\lambda \rightarrow 0} \lambda \mu(\{x \in X: M(f)(x)>\lambda\})
$$

exists but does not equal $\|f\|_{1}$ for $f \in L^{1}\left(\mathbb{R}^{n}, \mu\right)$. The reader may find examples for which even the above limit does not exist for doubling metric measure spaces. 


\section{ACKNOWLEDGMENT}

The first author was supported by a Major Basic Research Grant of NSFC (Grant No. 10631040).

\section{ReFERENCES}

[1] J. Heinonen, Lectures on analysis on metric spaces, Springer, 2001. MR1800917 (2002c:30028)

[2] P. Janakiraman, Limiting weak-type behavior for singular integral and maximal operators, Trans. Amer. Math. Soc. 358 (2006), 1937-1952. MR2197436 (2006m:42023)

[3] E.M. Stein, Harmonic Analysis: Real-Variable Methods, Orthogonality and Oscillatory Integrals, Princeton University Press, Princeton, New Jersey, 1993. MR1232192 (95c:42002)

[4] E.M. Stein, Singular integrals and differentiability properties of functions, Princeton University Press, 1970. MR0290095 (44:7280)

Department of Mathematical Sciences, Tsinghua University, Beijing 100084, People's Republic of China

E-mail address: hujiaxin@mail.tsinghua.edu.cn

Department of Mathematical Sciences, Tsinghua University, Beijing 100084, People's Republic of ChinA

E-mail address: hxp@mails.thu.edu.cn 